\title{
SPECTRA OF INFINITE GRAPHS VIA SCHUR COMPLEMENT
}

\section{GOLINSKII}

Abstract. The goal of the paper is to apply the general operator theoretic construction known as the Schur complement for computation of the spectrum of certain infinite graphs which can be viewed as finite graphs with rays attached to them. The examples of a multiple star and a flower with infinite rays are considered.

Mathematics subject classification (2010): 05C63, 05C76, 47A10, 47B15.

Keywords and phrases: Infinite graphs, adjacency operator, spectrum, block matrices, Green function.

\section{REFERENCES}

[1] M. Birman and M. Solomjak, Spectral Theory of Self-Adjoint Operators in Hilbert Spaces, D. Reidel Publishing Company, Dordrecht, 1987.

[2] A. E. Brouwer And W. H. Haemers, Spectra of Graphs, Springer, Universitext, 2012.

[3] F. CHung, Spectral graph theory, CBMS Regional Conference Series in Mathematics 92, Washington, 1997.

[4] D. M. CvetKović, M. Doob, AND H. SACHS, Spectra of Graphs - Theory and Applications, VEB Deutscher Verlag der Wissenschaften, Berlin, 1980.

[5] L. Golins KII, Spectra of infinite graphs with tails, Linear and Multilinear Algebra 64, 11 (2016), 2270-2296.

[6] R. Killip And B. Simon, Sum rules for Jacobi matrices and their applications to spectral theory, Ann. Math. 158 (2003), 253-321.

[7] V. LEBID AND L. NiZHNiK, Spectral analysis of locally finite graphs with one infinite chain, Proc. Ukranian Academy of Sci. 3 (2014), 29-35.

[8] V. LEBID AND L. NIZHNIK, Spectral analysis of certain graphs with infinite chains, Ukr. J. Math. 66 9 (2014), 1193-1204.

[9] B. MohaR, The spectrum of an infinite graph, Linear Alg. Appl. 48, 3 (1982), 245-256.

[10] B. Mohar And W. Woess, A survey on spectra of infinite graphs, Bull. London Math. Soc. 21, 2 (1989), 209-234.

[11] L. P. NiZhniK, Spectral analysis of metric graphs with infinite rays, Methods of Func. Anal. and Topology 20, 6 (2014), 391-396.

[12] I. SCHUR, Über Potenzreihen, die im Innern des Einheitskreises beschränkt sind, I, J. Reine Angew. Math. 147 (1917), 205-232.

[13] A. SCHWENK, Computing the characteristic polynomial of a graph, Graphs and combinatorics. Lect. Notes Math. 406 (1974), 153-172. 